\section{The use of toceranib phosphate after surgical resection of haemangiosarcoma from the aortic arch in a German Shepherd dog}

\section{Petra Černá ${ }^{1,2}$, Liz Welsh' ${ }^{1}$, Pamela Martin ${ }^{1}$ \\ 1 Vets Now Emergency and Referral Hospital Glasgow, Glasgow, United Kingdom \\ 2 University of Veterinary and Pharmaceutical Sciences Brno, Brno, Czech Republic}

\section{OBJECTIVES}

To report the post-operative outcome and survival of a dog after surgical resection of a haemangiosarcoma from the aortic arch using toceranib phosphate as an adjunctive therapy.

\section{METHODS}

Case report of a single patient treated with metronomic chemotherapy instead of conventional high-dose chemotherapy for adjuvant therapy of hemangiosarcoma of the aortic wall.

\section{RESULTS}

A 7-year-old male German Shepherd dog presented for investigation of lethargy. Pleural, peritoneal and pericardial effusion were identified, and echocardiography was indicative of a heart-based mass. The mass was surgically excised from the aortic arch and a subtotal pericardiectomy performed. Histology confirmed the mass was an incompletely excised haemangiosarcoma. The patient was started on epirubicin protocol $\left(25 \mathrm{mg} / \mathrm{m}^{2}\right)$ but developed a severe septic neutropenia and thrombocytopenia. This recurred following the second treatment of epirubicin despite a dose reduction of $10 \%$. Consequently, the patient was started on metronomic chemotherapy with toceranib phosphate $(80 \mathrm{mg}$ orally Monday, Wednesday and Friday weekly). Eight months following the diagnosis the patient remains systemically well on metronomic chemotherapy with an excellent quality of life. Repeat staging has been negative for local and distant metastases but shows tissue thickening at the level of the aortic arch.

\section{STATEMENT (CONCLUSIONS)}

Metronomic chemotherapy has been proposed for adjuvant therapy in dogs that do not tolerate conventional anthracycline based chemotherapy protocols. This is the first reported case of the use of a tyrosine kinase inhibitor as an adjuvant therapy for cardiac haemangiosarcoma.

\section{Hemipelvectomy in a rabbit: surgical technique and outcome}

\section{Laura Homer, Nicholas Bacon}

Fitzpatrick Referrals Oncology \& Soft Tissue, Guildford United Kingdom

\section{OBJECTIVES}

To describe the clinical presentation, surgical technique and postoperative outcome of a rabbit after a right-sided hemipelvectomy to treat a proximal femoral sarcoma.

\section{METHODS}

A seven-year-old female rabbit was presented with a sixmonth history of chronic right pelvic limb lameness. Radiographs revealed a lytic coxofemoral lesion and computerized tomography detailed an expansile lesion within the proximal femur, with an appearance consistent with a soft tissue mass. Incisional biopsies revealed a poorly differentiated sarcoma. A right-sided hemipelvectomy was performed. The pubis was cut paramedian at the cranial and caudal edges of the right obturator foramen and the sacroiliac joint was disarticulated. The resultant defect in the pelvic diaphragm was repaired by opposing the thoracolumbar fascia with external abdominal oblique and rectus abdominus with polydioxanone. Histopathology confirmed the biopsy findings, with tumor free margins achieved.

\section{RESULTS}

The patient recovered well from surgery and had good mobility. The patient survived 21 months post-surgery and died of non-cancer related disease.

\section{STATEMENT (CONCLUSIONS)}

To the authors' knowledge this is the first report of a hemipelvectomy performed in a rabbit. We describe the anatomical dissection in a cadaver to aid future surgeries Hemipelvectomy is a surgery that is more routinely performed in canine and feline patients, but with the right candidate and owner commitment to aftercare, it can safely and successfully be performed in rabbits. 